\title{
Emerging role of autophagy during ischemia-hypoxia and reperfusion in hepatocellular carcinoma
}

\author{
HAILEI DU ${ }^{1 *}$, WEIPING YANG ${ }^{2 *}$, LIN CHEN $^{3}$, BAIYONG SHEN $^{2}$, CHENGHONG PENG $^{2}$, \\ HONGWEI LI ${ }^{2}$, DAVID K. ANN ${ }^{4,5}$, YUN YEN ${ }^{4,5}$ and WEIHUA QIU ${ }^{2}$ \\ Departments of ${ }^{1}$ Thoracic Surgery, ${ }^{2}$ Surgery and ${ }^{3}$ Respiratory Disease, Ruijin Hospital, Shanghai Jiao Tong University \\ School of Medicine, Shanghai 200025, P.R. China; ${ }^{4}$ Department of Molecular Pharmacology and ${ }^{5}$ Irell and Manella \\ Graduate School of Biological Sciences, Beckman Research Institute, City of Hope, Duarte, CA 91010, USA
}

Received December 26, 2011; Accepted February 9, 2012

DOI: 10.3892/ijo.2012.1415

\begin{abstract}
Hepatocellular carcinoma (HCC) is the most common primary malignancy found in the liver. Autophagy is the intracellular bulk degradation process for long-lived proteins and dysfunctional organelles. In this study, we report that autophagy plays a role in HCC cell proliferation in response to ischemia-hypoxia $(\mathrm{I} / \mathrm{H})$ and reperfusion and discuss its potential therapeutic implications. By establishing a simulated model in cultured HepG2 (p53 wild-type) and Hep3B ( $p 53$ null) hepatoma cells in vitro, we found that exposure to $\mathrm{I} / \mathrm{H}$ induced a significant increase in microtubule-associated protein 1 light chain 3 (LC3) lipidation and subsequent LC3 puncta formation. While the proliferation of HCC cells was stimulated upon acute $\mathrm{I} / \mathrm{H}$ exposure compared to that of control, inhibition of autophagy by autophagy-related protein 7 interference abolished it. In addition, the steady-state levels of sequestosome 1 (p62) in both HepG2 and Hep3B cells were reduced following I/H exposure, supporting the notion that acute $\mathrm{I} / \mathrm{H}$ induces autophagy. Intriguingly, the p62 level further decreased during reperfusion following I/H, accompanied by increased LC3 lipidation. The intracellular reactive oxygen species (ROS) accumulated during acute $\mathrm{I} / \mathrm{H}$ exposure and persisted through reperfusion in both HepG2 and Hep3B cells and the ROS levels increased at a much faster rate during reperfusion than during $\mathrm{I} / \mathrm{H}$ periods in both
\end{abstract}

Correspondence to: Dr Weihua Qiu, Department of Surgery, Ruijin Hospital, Shanghai Jiao Tong University School of Medicine, Shanghai Institute of Digestive Surgery, 197 Ruijin Er Rd., Shanghai 200025, P.R. China

E-mail: drqwh2003@hotmail.com

Abbreviations: $\mathrm{I} / \mathrm{H}$, ischemia-hypoxia; $\mathrm{I} / \mathrm{N}$, ischemia-normoxia; Atg7, autophagy-related protein 7; LC3, microtubule-associated protein 1 light chain 3; p62, sequestosome 1; ROS, reactive oxygen species; CCK8, cell counting kit-8; HCC, hepatocellular carcinoma; NAC, $\mathrm{N}$-acetylcysteine

*Contributed equally

Key words: hepatocellular carcinoma, ischemia, hypoxia, reperfusion, autophagy cells. Autophagy functions as a promoter for HCC cell survival during acute $\mathrm{I} / \mathrm{H}$ and reperfusion and this also points to potential therapy for hepatoma by perturbing the acute I/H-reperfusionautophagy axis.

\section{Introduction}

Hepatocellular carcinoma (HCC) causes almost half a million deaths each year, making HCC the third most common cause of cancer-related mortality (1). Despite recent progress in diagnostic and therapeutic management, HCC prognosis remains poor (2). HCC is often diagnosed at an advanced stage when resection, transplantation, percutaneous and transarterial interventions are often of limited efficacy. Although sorafenib has demonstrated promising results in improving the survival and the time to progression in patients with advanced HCC, $\mathrm{HCC}$ is frequently resistant to conventional chemotherapy and radiotherapy (3).

Ischemia-hypoxia $(\mathrm{I} / \mathrm{H})$ and reperfusion constitute key components of many pathological conditions associated with solid tumors. As the liver is a key metabolic organ in humans, the abnormal vasculature network, high interstitial pressure and high proliferation rate of HCC cells result in different types of hypoxia, such as transient hypoxia and chronic hypoxia (4). In response to $\mathrm{I} / \mathrm{H}$, the formation of tumor collateral vessels can induce subsequent reperfusion, resulting in oxidative stress (5). Consistent with the described scenario, HCC cells are reportedly more sensitive to $\mathrm{I} / \mathrm{H}$ and reperfusion injury than normal liver tissue (6).

Transcatheter arterial chemoembolization (TACE) has become one of the most widely used treatments for patients with HCC as a palliative measure (7). However, increased microvessel density and vascular endothelial growth factor expression, resulting from $\mathrm{I} / \mathrm{H}$ following TACE, may cause limitations in therapeutic efficacy $(8,9)$. Moreover, the interplay of other compensatory cellular events against $\mathrm{I} / \mathrm{H}$ further complicate HCC growth, resistance, recurrence and metastasis.

Autophagy is a dynamic process in which cytosolic longlived proteins and pathological organelles are sequestered in double membrane vesicles (autophagosomes) (10). While autophagy appears to maintain cellular homoeostasis and facilitate adaptive responses to adverse conditions, it also 
modulates cell survival, differentiation, and growth as well as cellular defense (11). Results from other studies have suggested that the autophagic activity is higher in HCC tissues than that in normal liver (12). It has been proven that autophagic capacity relates to the progression of astrocytic tumors (13); however, to date, it remains to be elucidated whether autophagy is required for HCC survival, or whether it leads to HCC cell death, and is detrimental when hepatoma cells undergo $\mathrm{I} / \mathrm{H}$ and reperfusion. Thus, illuminating the role of autophagy and its underlying mechanisms in either enhancing cell survival or sensitizing cell death during hepatic $\mathrm{I} / \mathrm{H}$ and reperfusion would have a significant impact on treatment of HCC. In this study, HCC HepG2 ( $p 53$ wild-type) and Hep3B (p53 null) cells were employed i) to establish an $\mathrm{I} / \mathrm{H}$ and reperfusion model in vitro; ii) to determine autophagic activities and their role in promoting HCC cell survival during $\mathrm{I} / \mathrm{H}$ and reperfusion; and iii) to explore the correlation between p53 and autophagy induction in HCC upon $\mathrm{I} / \mathrm{H}$ exposure and reperfusion.

\section{Materials and methods}

Simulation of I/H and reperfusion. The human hepatoma cell lines, HepG2 (ATCC no: HB-8065) and Hep3B (ATCC no: HB-8064) were obtained from ATCC and cultured at $37^{\circ} \mathrm{C}$ in a humidified atmosphere of $95 \%$ air and $5 \% \mathrm{CO}_{2} . \mathrm{I} / \mathrm{H}$ and reperfusion models were established as described by HamacherBrady et al (14). Glucose deprivation in vitro was utilized to avoid inhibition of hypoxic response (15). Twenty-four hours after plating, ischemia was introduced via ischemia-mimetic solution (glucose deprivation solution) $(\mathrm{pH} 6.6,125 \mathrm{mM} \mathrm{NaCl}$, $8 \mathrm{mM} \mathrm{KCl}, 1.2 \mathrm{mM} \mathrm{KH}_{2} \mathrm{PO}_{4}, 1.25 \mathrm{mM} \mathrm{MgSO}_{4}, 1.2 \mathrm{mM} \mathrm{CaCl}_{2}$, $6.25 \mathrm{mM} \mathrm{NaHCO}, 5 \mathrm{mM}$ sodium lactate and $20 \mathrm{mM}$ HEPES) (16). In addition to being cultured in ischemia-mimetic solution, cells were simultaneously exposed to $1 \% \mathrm{O}_{2}$ (I/H group). $\mathrm{HCC}$ cells treated with ischemia-mimetic solution while being exposed to normoxic conditions were included as the control for hypoxia [ischemia-normoxia ( $\mathrm{I} / \mathrm{N})$ group]. Two hours after $\mathrm{I} / \mathrm{H}$ treatment, reperfusion was initiated by a buffer exchange to normoxic Krebs-Henseleit solution ( $\mathrm{pH} 7.4,110 \mathrm{mM} \mathrm{NaCl}$, $4.7 \mathrm{mM} \mathrm{KCl}, 1.2 \mathrm{mM} \mathrm{KH} \mathrm{PO}_{4}, 1.25 \mathrm{mM} \mathrm{MgSO}, 1.2 \mathrm{mM}$ $\mathrm{CaCl}_{2}, 25 \mathrm{mM} \mathrm{NaHCO}, 15 \mathrm{mM}$ glucose and $20 \mathrm{mM}$ HEPES). The cells were then incubated at $95 \%$ air and $5 \% \mathrm{CO}_{2}$ and harvested at $0,30,60,90,120$ and $180 \mathrm{~min}$ after reperfusion. The controls were exposed to DMEM solution before being exposed to normoxic conditions, and harvested at the corresponding time-points in a parallel manner.

Inhibition of autophagy by RNA interference. In order to inhibit autophagy specifically, autophagy-related protein 7 (Atg7) RNA interference was accomplished by transfecting HepG2 and Hep3B cells with siRNA. The Atg7-targeting sense sequence and the universal negative control siRNA were purchased from Invitrogen (Carlsbad, CA, USA) (12935-400). The human Atg7 sequence (5'-GGAGTCACAGCTCTTCCTT-3') was cloned into the BamH1 and EcoR1 sites of the pGSU6-GFP vector (Genlantis, San Diego, CA, USA). Control scrambled and shRNA plasmids were transfected into HepG2 and Hep3B cells plated at $50 \%$ confluence in six-well plates using Lipofectamine 2000 (Invitrogen). Forty-eight hours after transfection, cells were washed with PBS and collected for flow cytometry. Before being treated with ischemia-mimetic solution and incubated under hypoxic conditions, the cells were cultured for a further $24 \mathrm{~h}$.

Colorimetric viability assay. HepG2 and Hep3B cells were plated at a density of 5,000 cells/well in a volume of $100 \mu \mathrm{l}$ in 96-well microtiter plates and incubated overnight. The culture medium was removed the following day, and $100 \mu \mathrm{l}$ ischemia-mimetic solution were immediately added into each well. The plates were then incubated under hypoxic conditions as mentioned above. After 2-h maintenance under the $\mathrm{I} / \mathrm{H}$ conditions, the cells were administrated with $10 \mu \mathrm{l}$ cell counting kit 8 (CCK8) solution (Dojindo Laboratories, Japan) and incubated in a humidified $5 \% \mathrm{CO}_{2}$ atmosphere at $37^{\circ} \mathrm{C}$ for an additional $3 \mathrm{~h}$. The absorbance of the solution was read spectrophotometrically at $450 \mathrm{~nm}$ with a reference at $650 \mathrm{~nm}$ using a microtiter plate reader (Becton-Dickinson). The cell proliferation rate was calculated according to the following formula: cell proliferation $(\%)=[(\mathrm{A} 450$ sample-A450 blank $) /$ (A450 control - A450 blank) -1] x 100.

Immunofluorescent observation for microtubule-associated protein (MAP)1 light chain 3 (LC3)-positive autophagic vacuoles. After I/H exposure, HepG2 and Hep3B cells were fixed with $4 \%$ paraformaldehyde for $30 \mathrm{~min}$ and made permeable with methanol at $-20^{\circ} \mathrm{C}$ for $10 \mathrm{~min}$. Cells were blocked using $10 \%$ goat serum at room temperature for $30 \mathrm{~min}$. The cells were then incubated with rabbit polyclonal anti-LC3 (Santa Cruz Biotechnology, Santa Cruz, CA, USA) and mouse mononclonal anti-sequestosome 1 (p62) SQSTM1 antibodies (Santa Cruz Biotechnology). Goat anti-rabbit IgG-FITC and anti-mouse IgG-TR secondary antibodies were employed for fluorescent coloration. Immunofluorescent images were collected using a fluorescence microscope (Olympics IX71).

Detection of acidic vesicular organelles (AVOs) with acridine orange staining. Cells were treated with ischemia-mimetic solution and incubated under hypoxic conditions as described above. Acridine orange (Sigma) was added to the culture medium at a final concentration of $1 \mu \mathrm{g} / \mathrm{ml}$ post $\mathrm{I} / \mathrm{H}$ exposure and followed by incubation in the dark at room temperature for $15 \mathrm{~min}$. Samples were then examined under a fluorescence microscope. A typical acridine orange accumulation in acidic autophagic vacuoles exhibited granular distribution of bright red fluoresce in the cytoplasm, indicative of autophagosome formation. AVO fluorescence was measured with an excitation wavelength of $488 \mathrm{~nm}$ and an emission wavelength of $515 \mathrm{~nm}$. The amount of AVOs was quantitatively determined according to the red-to-green fluorescence ratio, which was obtained using Photoshop software (Adobe, San Jose, CA, USA).

Quantitative real-time RT-PCR. Steady-state LC3 mRNA levels were determined by real-time RT-PCR using SYBR Premix Ex Taq (Takara, Japan). Isolation of total RNA and reverse transcription of cDNA was performed as described previously (17). GAPDH was used as an endogenous control for the normalization of the differences in the amounts of total RNA in each sample. The primers pairs utilized were: LC3, 5'-TACGAGCAGGAGAAAGACGAGG-3' and 5'-GGCAGAG TAGGTGGGTTGGTG -3'; and GAPDH, 5'-GAAGGTGAA GGTCGGAGTC-3' and 5'-GAAGATGGTGATGGGAT 
TTC-3'. Thermal cycling was performed with the 7000 Sequence Detection System (Applied Biosystems, Carlsbad, CA, USA). Reactions were carried out in a $20-\mu 1$ reaction volume. PCR amplification conditions were as follows: $95^{\circ} \mathrm{C}$ for $10 \mathrm{~min}$, 40 cycles of $95^{\circ} \mathrm{C}$ for $5 \mathrm{sec}$ and $60^{\circ} \mathrm{C}$ for $30 \mathrm{sec}$. The cycle threshold $(\mathrm{Ct})$ data were obtained automatically and each data point was performed in triplicate.

Isolation of proteins and immunoblot analyses. Cells were harvested at various time-points by centrifugation at $800 \mathrm{rpm}$ for $5 \mathrm{~min}$ at $4^{\circ} \mathrm{C}$. The isolation of proteins, electrophoresis, and the polyvinylidene difluoride membranes transfer were performed as described previously (18). Blots were probed with rabbit polyclonal anti-LC3, mouse monoclonal anti-HIF- $\alpha$ (Santa Cruz Biotechnology), mouse monoclonal anti-p62, mouse monoclonal anti-GAPDH antibodies (Santa Cruz Biotechnology), and rabbit monoclonal Atg7 antibody (Novus Biologicals, Littleton, CO, USA) overnight at $4^{\circ} \mathrm{C}$. GAPDH was used as the internal control. A goat anti-rabbit (Santa Cruz Biotechnology) or goat anti-mouse IgG secondary antibody (Santa Cruz Biotechnology) was incubated at room temperature for $1 \mathrm{~h}$. The blots were then incubated with chemiluminescent substrate and detected by enhanced chemiluminescence assay (Millipore). Band density was measured by densitometry, quantified using Photoshop software, and normalized to an indicated sample in the identical membrane.

Determination of intracellular reactive oxygen species (ROS). The generation of ROS in the cells was evaluated by a fluorometry assay using intracellular oxidation of the non-fluorescent probe 2,7-dichlorofluorescein diacetate (DCFH-DA). DCFH-DA can passively diffuse into cells and be deacetylated by esterase to form non-fluorescent 2,7-dichlorofluorescein (DCFH). In the presence of ROS, DCFH reacts with ROS to form the fluorescent product, DCF, which is trapped inside the cells. When the membrane is oxidized and damaged, the fluorescence will attenuate significantly. The $\mathrm{I} / \mathrm{H}$ and reperfusion treatment was performed as described above. The cells were washed with ice-cold 1X PBS and then incubated with DCFH-DA at $37^{\circ} \mathrm{C}$ for $20 \mathrm{~min}$. DCF fluorescence intensity was then detected by fluorescence spectrometry (Spectramax Gemini, Molecular Devices, Sunnyvale, CA, USA) with an excitation wavelength of $490 \mathrm{~nm}$ and an emission wavelength of $530 \mathrm{~nm}$. The results were expressed as relative fluorescence intensity per $10^{4}$ cells. N-acetylcysteine (NAC; Sigma), the specific inhibitor of ROS, was further employed to confirm the specific generation of ROS during reperfusion. Two hours before reperfusion exposure, the cells were pretreated with NAC at a final concentration of $5 \mathrm{mM}$.

Statistical analysis. Statistical analysis was performed using SPSS software (v.13.0). Data are expressed as the means \pm SD of three individual experiments. Comparisons of quantitative data were analyzed using the Student's t test between two groups or by one-way ANOVA for multiple groups. We assumed statistically significant differences at $\mathrm{p}<0.05$.

\section{Results}

The confirmation of $I / H$ and reperfusion model in vitro. We developed an experimental paradigm of utilizing the ischemia- mimetic solution coupled with $1 \% \mathrm{O}_{2}$ hypoxic exposure for $\mathrm{I} / \mathrm{H}$ exposure and the following re-oxygenation and glucose replenishment with normoxic Krebs-Henseleit solution for reperfusion. Hypoxia induced factor- $1 \alpha(\mathrm{HIF}-1 \alpha)$ is a major hypoxia-induced transcriptional factor. In normoxia, the steady-state HIF-1 $\alpha$ protein level was very low due to a rapid degradation via the ubiquitin-proteasome pathway. Upon hypoxic exposure, HIF-1 $\alpha$ protein was stabilized and translocated to the nucleus for transcriptional activation of hypoxia-responsive genes (19). As expected, the mean ratio of HIF-1 $\alpha$ to GAPDH increased significantly at $2 \mathrm{~h}$ post $\mathrm{I} / \mathrm{H}$ exposure compared with the mock exposure in both HepG2 and Hep3B cells (HepG2: 0.23 \pm 0.01 versus $0.06 \pm 0.01, \mathrm{p}<0.01$; Hep3B: $0.14 \pm 0.00$ versus $0.06 \pm 0.01$, $\mathrm{p}<0.01)$. By contrast, the cells under $\mathrm{I} / \mathrm{N}$ conditions failed to exhibit an increase in HIF- $1 \alpha$ expression $\left(\mathrm{p}_{\mathrm{HepG} 2}=0.599\right.$, $\left.\mathrm{p}_{\mathrm{Hep3B}}=0.474\right)$, confirming the validity of the $\mathrm{I} / \mathrm{H}$ model.

Activation of autophagy in HCC cells during I/H. In a previous study, we reported that acute hypoxic stress induces autophagy in normal cells (20). As shown in Fig. 1A, the formation of LC3-positive puncta increased in both HepG2 and Hep3B cells at $2 \mathrm{~h}$ post $\mathrm{I} / \mathrm{H}$-treatment. Living cell staining with acridine orange revealed a significant increase in the red-to-green fluorescence ratio (AVO accumulation) in both $\mathrm{I} / \mathrm{H}$-stressed HepG2 and Hep3B cells compared to the control. By contrast, there were relatively few AVOs in the cytoplasm of cells of the control and $\mathrm{I} / \mathrm{N}$ groups (Fig. 1B). Although a slight increase in the ratio was noticed in the cells of the I/N group, no statistical significance was rendered. These data show that acute $\mathrm{I} / \mathrm{H}$ exposure, but not ischemia alone, induces autophagy in both HepG2 and Hep3B cells.

Quantitative real-time RT-PCR analysis showed that the mean ratios of $L C 3$ to $G A P D H$ mRNA significantly decreased from $2.08 \pm 0.21$ in the control group to $1.26 \pm 0.44$ in the HepG2 cells at $2 \mathrm{~h}$ post $\mathrm{I} / \mathrm{H}$ exposure $(\mathrm{p}=0.018)$ (Fig. 1C). By contrast, steady-state LC3 mRNA levels in the Hep3B cells increased significantly from $3.91 \pm 1.06$ in the control group to $11.75 \pm 2.33$ following $\mathrm{I} / \mathrm{H}$ treatment $(\mathrm{p}=0.001)$. HepG2 cells, lacking AVO formation, exhibited the apparent decrease of $L C 3$ mRNA post $\mathrm{I} / \mathrm{N}$ exposure, as observed with $\mathrm{I} / \mathrm{H}$. The opposite change in $L C 3$ mRNA levels in these two HCC cell lines raises the possibility that p53 may fine-tune autophagy activation through the differential transcriptional control of LC3 in response to $\mathrm{I} / \mathrm{H}$.

Western blot analysis on LC3 lipidation (LC3-II) is regarded as a useful means to monitor autophagy (21). As shown in Fig. 1D, LC3 lipidation increased markedly at $2 \mathrm{~h}$ post $\mathrm{I} / \mathrm{H}$ exposure. Quantitative analyses revealed that the post-I/H LC3 lipidation increased significantly from $0.15 \pm 0.01$ to $0.37 \pm 0.03$ in HepG2 cells $(\mathrm{p}<0.01)$ and from $0.12 \pm 0.01$ to $0.36 \pm 0.04$ in Hep3B cells $(\mathrm{p}<0.01)$. While $\mathrm{I} / \mathrm{H}$ conveyed opposite changes on the LC3 mRNA levels, LC3 lipidation showed a comparable increase from acute $\mathrm{I} / \mathrm{H}$ in HepG2 and Hep3B cells. However, $\mathrm{I} / \mathrm{N}$ did not result in a statistically significant difference in LC3 lipidation in the HepG2 and Hep3B cells.

Effects of autophagy on HCC cell proliferation during acute $I / H$. Next, the effects of acute $\mathrm{I} / \mathrm{H}$ on $\mathrm{HCC}$ cell proliferation were evaluated. As an essential autophagy gene, Atg7 is an E1-like enzyme involved in the ubiquitination of MAP-LC3 early in the pathway (22); the knockdown of Atg7 can inhibit autophagy 
A
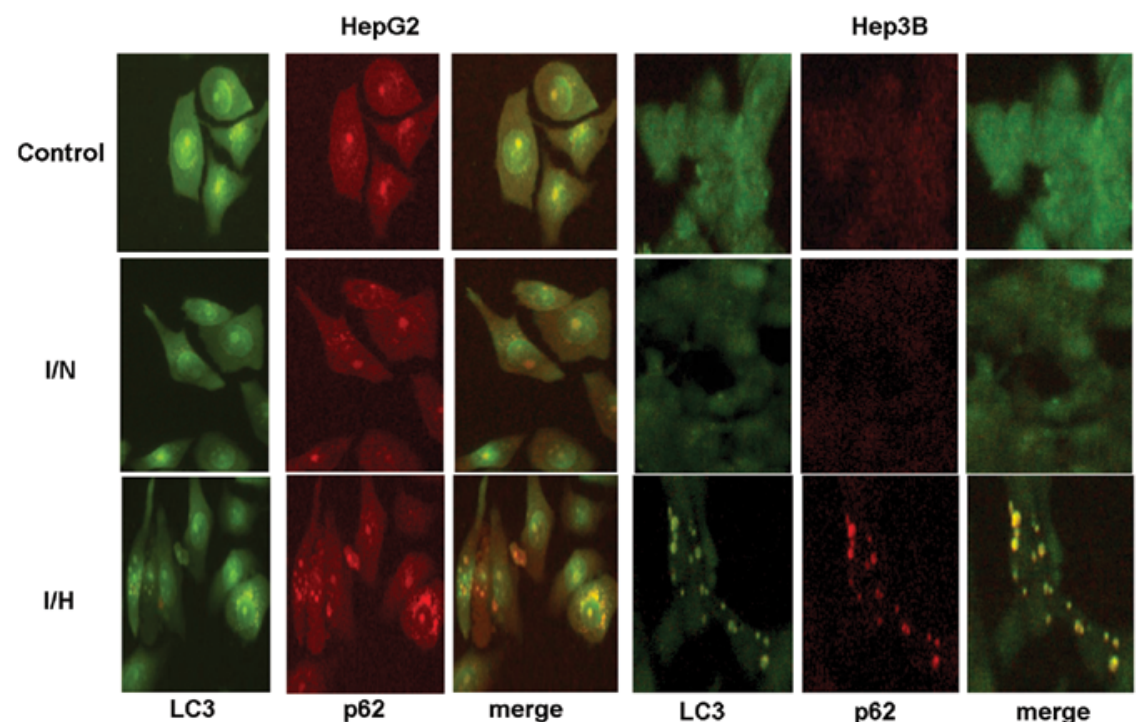

B



HepG2

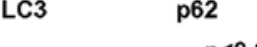

merge

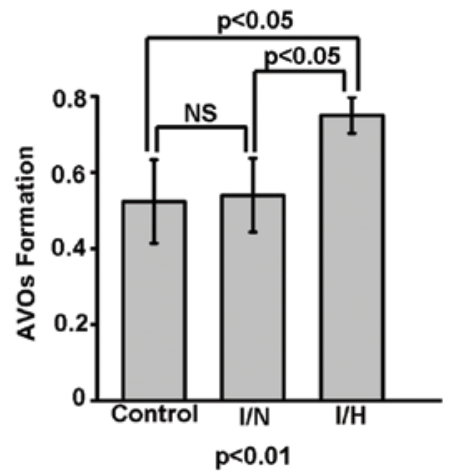

c

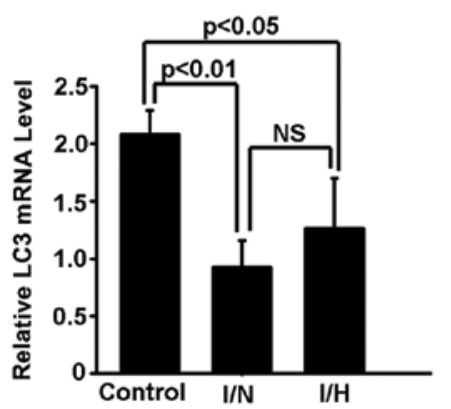

HepG2

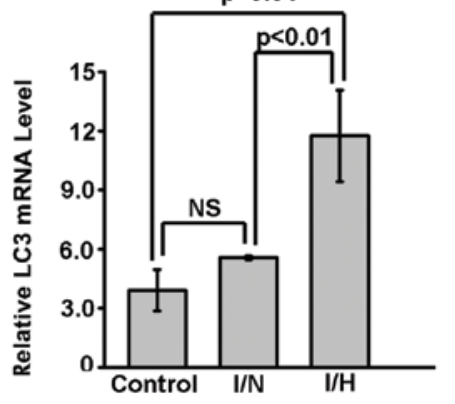

D
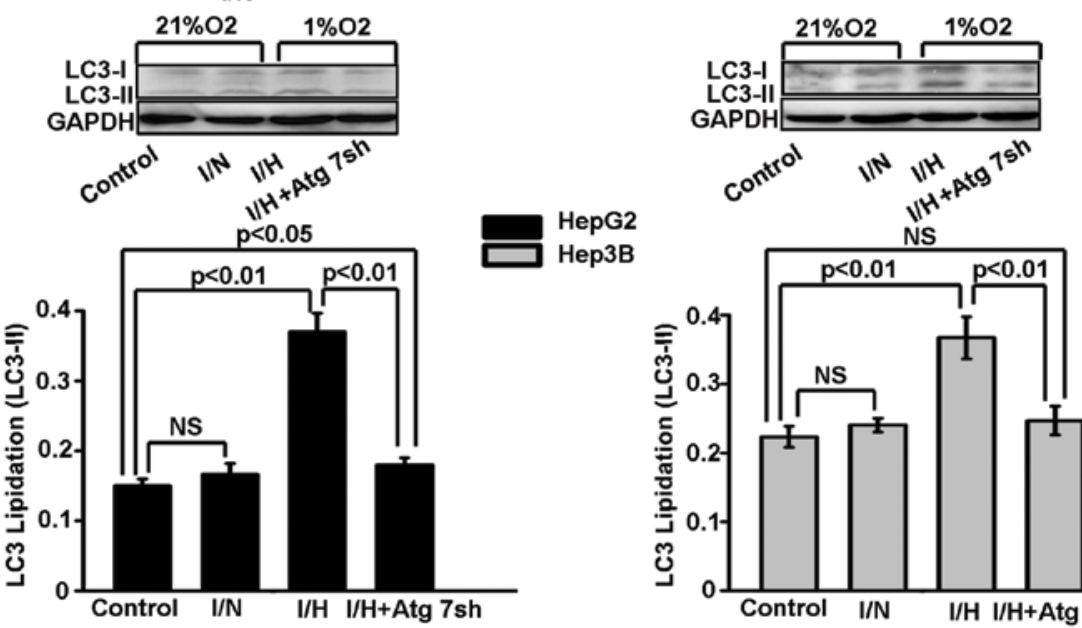

HepG2

Hep3B



Figure 1. Activation of autophagy in HepG2 and Hep3B by I/H treatment. I/H treatment in HepG2 and Hep3B was introduced via ischemia-mimetic solution and $1 \% \mathrm{O}_{2}$ exposure for $2 \mathrm{~h}$. (A) Formation of LC3-positive puncta. Cells were treated with I/N or I/H and the formation of autophagic vacuoles was determined by immunofluorescent staining for LC3 and p62. (B) Detection of AVOs with acridine orange staining. The formation of AVOs was quantified by the detection of red and green fluorescence after acridine orange staining. The red-to-green fluorescence ratio (AVOs) in both HepG2 and Hep3B cells under I/H treatment was compared to that in the same cells treated with DMEM (control) or I/N. (C) Steady-state LC3 mRNA levels were measured by quantitative real-time RT-PCR analyses. (D) LC3 lipidation was assessed by western blot analysis. The data shown here are representative of three independent experiments. Densitometry was performed using Photoshop and the results are presented as the means \pm SD. 
specifically. Therefore, the cells were pre-treated with Atg7 interference under nutrient-poor conditions for $1 \mathrm{~h}$ to assess the contribution of autophagy in promoting $\mathrm{HCC}$ cell proli-feration under $\mathrm{I} / \mathrm{H}$ and reperfusion. As shown in Fig. 2A, western blot analysis confirmed that Atg7 expression was inhibited by siRNA administration. Pre-treatment with Atg7 interference inhibited the LC3 lipidation increase induced by I/H (Fig. 1D). As shown in Fig. 2B, HepG 2 cells grew approximately $10 \%$ under the $\mathrm{I} / \mathrm{H}$ environment. A comparable stimulating effect was also observed in the Hep3B cells. Atg7 interference inhibited I/H-induced cell proliferation in the HepG2 and Hep3B cells. Cell proliferation decreased from $13.24 \pm 1.18 \%$ to $7.85 \pm 1.56 \%\left(\mathrm{p}_{\mathrm{HepG} 2}<0.01\right)$ and from $11.66 \pm 0.92 \%$ to $6.85 \pm 1.10 \%\left(\mathrm{p}_{\mathrm{Hep} 3 \mathrm{~B}}<0.001\right)$, in the HepG2 and Hep3B cells, respectively (Fig. 2B). More importantly, the Atg7 interference treatment alone had almost no inhibitory effect on the proliferation of HepG2 and Hep3B cells $\left(7.05 \pm 1.57 \%\right.$ versus $7.71 \pm 1.53 \%, \mathrm{p}_{\mathrm{HepG} 2}=0.578 ; 5.38 \pm 1.04 \%$ versus $\left.6.00 \pm 0.81 \%, \mathrm{p}_{\text {Нер } 3 \mathrm{~B}}=0.491\right)$. These results collectively suggest that the observed inhibitory effect by Atg7 interference on HCC cell proliferation following acute $\mathrm{I} / \mathrm{H}$ exposure most likely resulted from the inhibitory effect of Atg7 interference on autophagy.

We further explored the effect of acute $\mathrm{I} / \mathrm{H}$ exposure on the proliferation of HCC cell lines with different p53 contexts (p53 wild-type and p53 null). To eliminate the difference of growth character between HepG2 and Hep3B cells, the proliferation rate was compared according to the ratio of $\mathrm{I} / \mathrm{H}$ group to the control group. As shown in Fig. 2C, the proliferation ratio for Hep3B cells was slightly higher than that for HepG2 cells, and there was no significant difference between these two cells in response to acute $\mathrm{I} / \mathrm{H}(1.02 \pm 0.03$ versus $1.13 \pm 0.05, \mathrm{p}=0.247)$. When autophagy was inhibited by Atg7 knockdown, a minor difference in cell proliferation between HepG2 and Hep3B cells was noted $(1.01 \pm 0.03$ versus $1.13 \pm 0.05, \mathrm{p}=0.03)$. These results indicate the involvement of an Atg7-independent pathway in cell proliferation following $\mathrm{I} / \mathrm{H}$.

Changes in p62 expression following acute $I / H$. The p62 protein is a multifunctional signal adaptor protein that can be incorporated into the completed autophagosome and degraded in autolysosomes (23). We used p62 as a surrogate marker to monitor autophagic flux. As shown in Fig. 2D, the mean ratio of p62 to GAPDH in HepG2 cells decreased from $0.30 \pm 0.02$ to $0.12 \pm 0.01(\mathrm{p}<0.01)$ following $\mathrm{I} / \mathrm{H}$-treatment. A similar result was observed in Hep3B cells (from $0.11 \pm 0.01$ to $0.04 \pm 0.01$, $\mathrm{p}<0.01)$. Consistent with the results of the LC3 lipidation profile (Fig. 1D); there was no statistical difference in p62 levels between the I/N and control groups. Pre-treatment with Atg7 interference reversed the p62 decrease induced by $\mathrm{I} / \mathrm{H}$. The increased clearance of the p62 protein further confirms that acute $\mathrm{I} / \mathrm{H}$ stimulates human $\mathrm{HCC}$ cell growth resulting from autophagic activation.

Activation of autophagy in HCC cells during reperfusion. The role of autophagy in promoting HCC cell proliferation under I/H stress has been demonstrated; however, the function of autophagy during reperfusion remains unexplained. In our study, continuous autophagosomal accumulation was observed in both HepG2 and Hep3B cells during reperfusion (Fig. 3A). Quantitative analyses showed that reperfusion further increased LC3 lipidation in both HepG2 and Hep3B cells, compared with those under I/H conditions [Fig. 3B; HepG2: $(0-30 \mathrm{~min}) \mathrm{p}=0.896,(0-60 \mathrm{~min}) \mathrm{p}=0.025,(0-90 \mathrm{~min})$ $\mathrm{p}=0.003,(0-120 \mathrm{~min}) \mathrm{p}=0.248,(0-180 \mathrm{~min}) \mathrm{p}<0.01$; Hep3B: $(0-30 \mathrm{~min}) \mathrm{p}=0.488,(0-60 \mathrm{~min}) \mathrm{p}=0.094(0-90 \mathrm{~min}) \mathrm{p}<0.01$, $(0-120 \mathrm{~min}) \mathrm{p}<0.01,(0-180 \mathrm{~min}) \mathrm{p}<0.01]$. The $\mathrm{I} / \mathrm{H}$-induced autophagy persisted even during the reperfusion stage in HepG2 and Hep3B cells. Notably, HepG2 cells showed two apparent decreases in LC3 lipidation at the 60- and 120-min time-points post reperfusion. These results raise the possibility that p53 status also regulates autophagic activation during reperfusion. Thus, it is possible that $\mathrm{I} / \mathrm{H}$-triggered autophagy continues through reperfusion.

Changes in p62 expression during reperfusion. Fig. 3C shows that the p62 level significantly decreased following acute $\mathrm{I} / \mathrm{H}$ exposure as a result of the induced autophagic process. However, during reperfusion, the p62 level unexpectedly increased at 30 and 60 min post-reperfusion while autophagy was ongoing in HepG2 cells (0-30 min, $\mathrm{p}=0.009$; 0-60 min, $\mathrm{p}=0.006)$. This was followed by a decrease from $90-180 \mathrm{~min}$ post-reperfusion in HepG2 cells (0-90 min, $p=0.003 ; 0-120 \mathrm{~min}, \mathrm{p}=0.114 ; 0-180 \mathrm{~min}$, $\mathrm{p}=0.001)$. By contrast, a sustained decrease in the p62 level during reperfusion compared with that during $\mathrm{I} / \mathrm{H}$ was observed in Hep3B cells $[(0-30 \mathrm{~min}) \mathrm{p}<0.01,(0-60 \mathrm{~min}) \mathrm{p}<0.01,(0-90 \mathrm{~min})$ $\mathrm{p}<0.01,(0-120 \mathrm{~min}) \mathrm{p}<0.01,(0-180 \mathrm{~min}) \mathrm{p}<0.01)]$. The decrease in the steady-state $\mathrm{p} 62$ protein level further supports our hypothesis that autophagy continues to function during reperfusion.

ROS generation during $I / H$ and reperfusion exposure. To investigate whether the oxidative burst mediates the majority of the damage during $\mathrm{I} / \mathrm{H}$ and reperfusion exposure in HepG2 and Hep3B cells, intracellular ROS levels were measured. As shown in Fig. 4A and B, while the basal ROS level in Hep3B cells was relatively lower than that in HepG2 cells, ROS accumulated during acute $\mathrm{I} / \mathrm{H}$ exposure and persisted through reperfusion in both HepG2 and Hep3B cells. Notably, the ROS level increased at much faster rate during reperfusion than during $\mathrm{I} / \mathrm{H}$ periods in both cells. Pre-treatment with Atg7 interference decreased ROS accumulation in both HepG2 and Hep3B cells. When ROS was further specifically inhibited by NAC, the increased generation of ROS was ruled out in both cells. There was no difference in ROS accumulation when compared with that in the control ( $>0.05$ ). Thus, our results suggest that $\mathrm{I} / \mathrm{H}$ and reperfusion trigger ROS generation, which is consistent with the activation of autophagy.

\section{Discussion}

Along with the advancement of chemotherapy and intervention technology, TACE has become an important method in the treatment of unresectable HCC. However, clinical observations have demonstrated that rapid recurrence and metastasis, even after tumors are eradicated with TACE, are frequently detected in many patients. It is possible that HCC cells likely have a compensatory mechanism to enhance cell survival or promote cell proliferation in a hypoxic microenvironment. Hypoxiainduced angiogenesis and hexokinase II expression have been reported as part of compensatory mechanisms for resistance to the TACE procedure $(24,25)$. In this study, we report that 
A
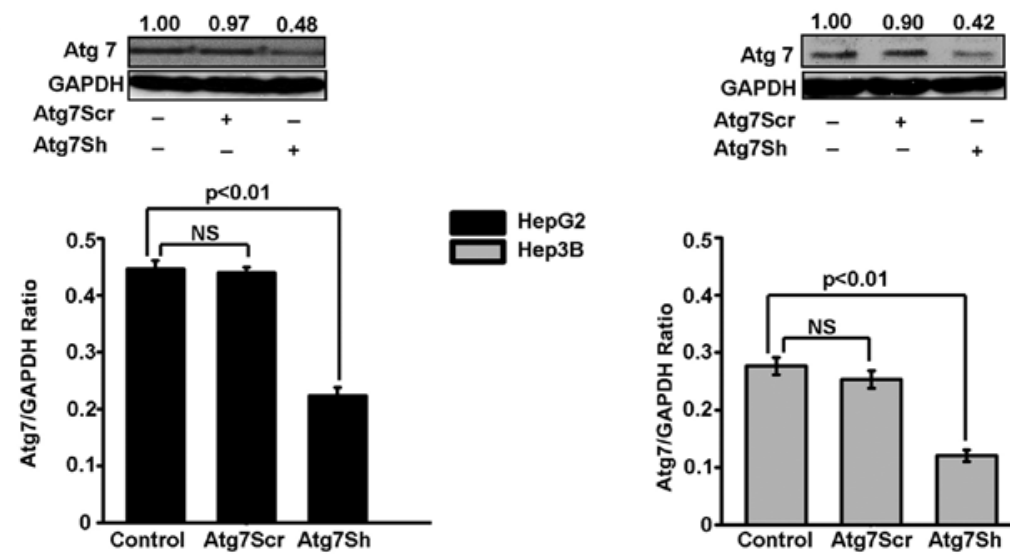
HepG2
Hep3B


D
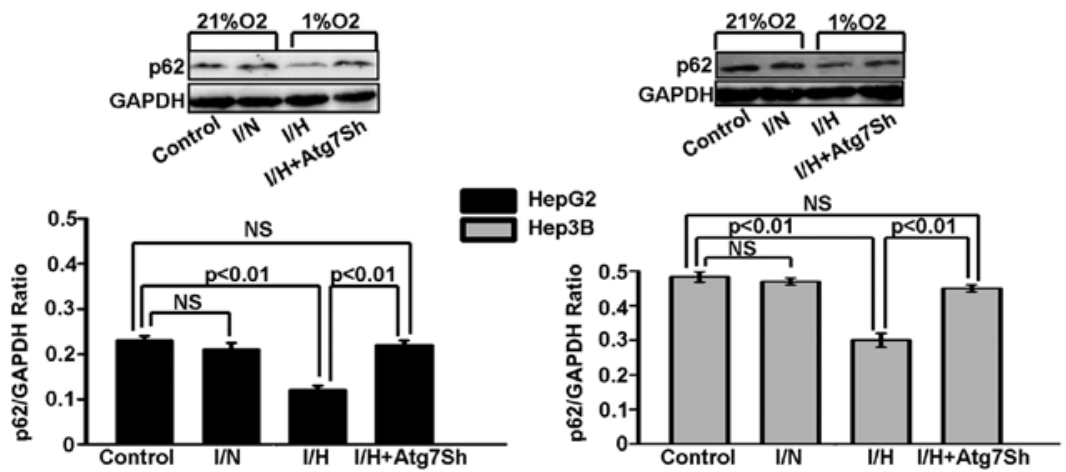

Figure 2. Effects of autophagy on HCC cell proliferation after I/H treatment. (A) Autophagy was specifically inhibited by Atg7 siRNA interference. The Atg7 knockdown was confirmed using western blot analysis. (B) Comparison of cell proliferation with or without autophagic inhibition. (C) Comparison of cell proliferation between HepG2 and Hep3B cells. To eliminate the difference in growth character between HepG2 and Hep3B cells, the proliferation rate was compared according to the ratio of the $\mathrm{I} / \mathrm{H}$ group to the control group. The left panel shows the cell proliferation rate after $\mathrm{I} / \mathrm{H}$ treatment and the right panel the cell proliferation rate with Atg7 interference and I/H exposure. (D) p62 accumulation after I/H treatment was measured by western blot analysis. Atg7Scr and Atg7Sh, cells transfected with control siRNA (scrambled) or Atg7 ShRNA; IH + Atg7Sh, Atg7 interference before I/H treatment; Atg7Sh, Atg7 interference without I/H treatment. Data represent the means \pm SD of three independent experiments.

acute $\mathrm{I} / \mathrm{H}$ and reperfusion induce autophagy and stimulate the proliferation of HCC HepG2 and Hep3B cells. This suggests that autophagic induction is an underlying mechanism for cells in HCC nodules to survive TACE treatment (26). 
A

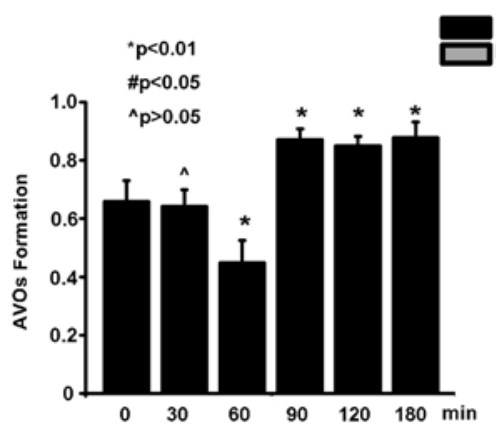

HepG2

Hер3В *p<0.01


Figure 3. Autophagic process during reperfusion. Two hours after $\mathrm{I} / \mathrm{H}$ treatment, reperfusion was initiated by a buffer exchanging to normoxic Krebs-Henseleit solution. The cells were then incubated at $95 \%$ air and $5 \% \mathrm{CO}_{2}$ and harvested at $0,30,60,90,120$ and 180 min after reperfusion. (A) Detection of AVOs with acridine orange staining during reperfusion after I/H treatment by fluorescence microscopy. (B) LC3 lipidation relative to GAPDH as the internal loading control was detected using western blot analysis. (C) The accumulation of p62 during reperfusion was measured by western blot analysis. The data shown here are representative of three independent experiments. Densitometric tracing was performed using Photoshop and the results are presented as the means \pm SD.

A



- I/H+R+NAC

$$
=1 / \mathrm{H}+\mathrm{R}
$$

$-1 / \mathrm{H}+\mathrm{R}+\mathrm{Atg} 7 \mathrm{Sh}$

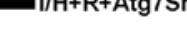

${ }^{*} \mathrm{p}<0.01$

$\# p<0.05$

\section{$\wedge p>0.05$}

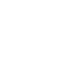

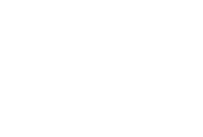



Нер3в

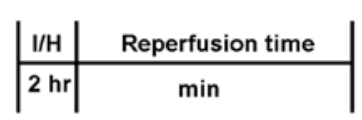

Figure 4. The specific generation of ROS during I/H and reperfusion. Intracellular ROS levels were measured to investigate whether the oxidative burst mediates the majority of the damage during I/H and reperfusion exposure. The ROS generations in HepG2 (A) and Hep3B (B) were measured by a fluorometry assay using intracellular oxidation of DCFH-DA and further confirmed by Atg7 interference and NAC inhibition. The comparison was carried out between data in different time-points and the control. 
Mechanistically, resistance to TACE can be construed as being primarily a resistance to $\mathrm{I} / \mathrm{H}$ commitment. Oxygendependent mitochondrial ATP generation is responsible for providing the energy required for cell proliferation and survival. In a hypoxic state, a distinct pathway is operated for ATP generation (27). For example, free fatty acids and amino acids from deprecated lipids and proteins from the autophagic process are re-used to maintain mitochondrial ATP production and protein synthesis as well as to promote cell survival (28). As autophagy could serve as an effective strategy to provide a temporary source of metabolic energy and catabolism intermediates (29), autophagic response is crucial for cell survival during pathologically relevant stresses, such as ischemia and hypoxia.

Based on the results shown in the present study, including the increased formation of LC3-positive autophagosomes (Fig. 1A), accumulation of AVOs and elevated LC3 lipidation upon exposure to I/H (Fig. 1D), we concluded that acute I/H-treatment induces autophagy in both HepG2 and Hep3B cells. Unexpectedly, ischemic treatment without hypoxia (I/N) failed to stimulate the autophagic process (Fig. 1). It is possible that the 2-h long I/N-treatment alone may be too short to trigger autophagic activation. Alternatively, as cancer cells, HepG2 and Hep3B cells may already have accumulated defects in their response to starvation. Nonetheless, we unequivocally demonstrated that both the HepG2 and Hep3B cells proliferated at a faster rate under acute $\mathrm{I} / \mathrm{H}$ conditions than those under normoxic conditions. While chronic hypoxia has been proven to alter the DNA repair pathway and drive genome instability and drug resistance (30), our observations further question the paradoxical role of acute hypoxia as a therapeutic target for the management of HCC.

The relationship between autophagy, tumorigenesis and progression has been extensively debated; while autophagy has been viewed as a tumor-suppressing mechanism, autophagy also contributes to the rapid tumor progression (31). Notably, the pro-survival function of autophagy may help cancer cells to survive in nutrient-limited environments and to resist ionizing radiation and chemotherapies (32). As shown in the present study, an autophagic inhibitor can suppress HCC cell growth during $\mathrm{I} / \mathrm{H}$, suggesting that $\mathrm{I} / \mathrm{H}$ is likely to initiate an autophagic response to promote $\mathrm{HCC}$ cell survival, potentially accounting for the increased HCC cell proliferation after TACE. Following ischemia, reperfusion, likely resulting from angiogenesis, collateral circulation establishment and other mechanisms, usually functions as a compensatory process. In our study, autophagy and LC3 lipidation displayed an overall increase in both HepG2 and Hep3B cells during the reperfusion phase after acute $\mathrm{I} / \mathrm{H}$. However, higher levels of autophagy induced by reperfusion may cause excessive self-digestion and eventual cell death. We postulate that response to $\mathrm{I} / \mathrm{H}$ and reperfusion, depending on the duration and intensity, may either enhance cell survival by autophagy or sensitize cell death by excessive autophagic activity.

p62/SQSTM1 is commonly found in protein aggregation diseases affecting both the brain and liver. Liver-specific autophagy defects in mice cause accumulation of p62 aggregates, oxidative stress and p62-dependent hepatocyte death (33). Previous findings have linked p62 activity to the extrinsic apoptotic pathway, suggesting that the modulation of $\mathrm{p} 62$ by autophagy is a key factor in carcinogenesis (34). The hypoxic clearance of p62 by autophagy plays an essential role in the protection of cancer cells against hypoxic stress (35). These findings place p62 at a critical position to control cell death and survival. In our study, the activation of autophagy by acute $\mathrm{I} / \mathrm{H}$ was accompanied with the increased clearance of $\mathrm{p} 62$. Consistent with a previous study (31), our results support the notion that autophagy-mediated clearance of p62 under acute $\mathrm{I} / \mathrm{H}$ promotes $\mathrm{HCC}$ cell survival. In addition, we found that the p62 protein level increased rapidly and was then followed by a decrease during reperfusion in HepG2 cells, while it continued to decrease in Hep3B cells. These results suggest that the availability of oxygen after $\mathrm{I} / \mathrm{H}$ differentially regulates the steady-state level of p62 in HepG2 and Hep3B cells. However, the exact pathophysiological implication of this observation needs to be further elucidated.

p53, the most extensively characterized tumor suppressor protein in mammalian cells, is activated after DNA damage, oncogene activation, hypoxia or metabolic alterations. The association between $p 53$ and autophagy is complex, as p53 appears to play a dual role in autophagic regulation (36). The mysteries underlying the p53-mediated regulation of autophagy extend beyond the question of whether p53-modulated autophagy is pro-death or pro-survival. For example, while nuclear p53 can induce autophagy through transcriptional effects, cytoplasmic p53 may act as a master repressor of autophagy (32). The distinct difference in p53 status between HepG2 ( $p 53$ wildtype) and Hep3B ( $p 53$ null) cells makes them a useful model to investigate the role of $\mathrm{p} 53$ in modulating $\mathrm{I} / \mathrm{H}$ - and reperfusioninduced autophagy. Our results indirectly suggest there is no difference in autophagy activation during $\mathrm{I} / \mathrm{H}$ regardless of p53 status. However, when autophagy was inhibited by Atg7 interference, the effect on subsequent cell proliferation was context-dependent. We further demonstrated that there were two apparent decreases in LC3 lipidation with a concomitant increase of p62 at 60 and 120 min post reperfusion, confirming that some feedbacks exist to inhibit active autophagy during reperfusion in HepG2 cells. By contrast, lack of p53 in Hep3B cells manifested more apparent autophagic parameters, such as LC3 lipidation, redistribution of LC3 in cytoplasmic puncta, accumulation of autophagosomes, and depletion of p62/ SQSTM1 during reperfusion. It has been reported that $p 53$-null cells are insensitive to metabolic stress with a higher intracellular ATP level (37). Our results provide additional evidence to support the possibility that $p 53$ null cells are better equipped with more active autophagy to survive metabolic stress.

Recent studies have suggested that reperfusion of ischemic tissue generates large amounts of ROS rapidly. There exists a positive correlation between the severity of cell injury and the amount of ROS generated. A rapid change in the mitochondrial complex redox states results in the leak of electrons to oxygen and a decreased availability of ADP. This induces ROS formation after ischemia and the subsequent re-introduction of oxygen during reperfusion. It has been reported that there exists a functional link between ROS generation, autophagy and apoptosis induction (38). In our study, ROS formation accumulated following acute $\mathrm{I} / \mathrm{H}$ exposure and continued to rapidly increase during reperfusion. The consistence of ROS generation and the activation of autophagy reveal that the initial ROS release may trigger autophagy as a protective defense mechanism of HCC cells to deal with $\mathrm{I} / \mathrm{H}$ and reperfusion exposure. 
Taken together, the results from our study suggest that the autophagic pathway plays a critical role in promoting HCC cell proliferation during $\mathrm{I} / \mathrm{H}$ and that the inhibition of autophagy, at least in part, effectively suppresses the accelerated cell proliferation during $\mathrm{I} / \mathrm{H}$ in a context-dependent manner. Consequently, the intermittent activation of autophagy as a survival strategy could accelerate the progression of HCC, potentially explaining the resistance of HCC to certain ablation therapies. Since the blockage of autophagy attenuates I/H-stimulated cell proliferation, the inhibition of autophagy could be considered as a therapeutic target for the management of HCC.

\section{Acknowledgements}

This study was supported by the Nature Science Foundation of China (81172326, 30872511), Shanghai Science and Technology Commission Grant (10ZR1419400) and Shanghai Charity Foundation for Cancer Research.

\section{References}

1. Caldwell S and Park SH: The epidemiology of hepatocellular cancer: from the perspectives of public health problem to tumor biology. J Gastroenterol 44 (Suppl 19): 96-101, 2009.

2. Olsen SK, Brown RS and Siegel AB: Hepatocellular carcinoma: review of current treatment with a focus on targeted molecular therapies. Therap Adv Gastroenterol 3: 55-66, 2010.

3. Roberts LR: Sorafenib in liver cancer - just the beginning. N Engl J Med 359: 420-422, 2008.

4. Uen YH, Ko PH, Yin PH, Liu TY, Chi CW and Lui WY: Glucocorticoid protects hepatoma cells against metabolic stressinduced cell death. Int J Oncol 33: 1263-1270, 2008.

5. Lencioni R: Loco-regional treatment of hepatocellular carcinoma in the era of molecular targeted therapies. Oncology 78 (Suppl 1): 107-112, 2010.

6. Choukèr A, Martignoni A, Schauer R, Dugas M, Rau HG, Jauch KW, Peter K and Thiel M: Beneficial effects of ischemic preconditioning in patients undergoing hepatectomy: the role of neutrophils. Arch Surg 140: 129-136, 2005.

7. Imai N, Ikeda K, Seko Y, Kawamura Y, Sezaki H, Hosaka T, et al: Previous chemoembolization response after transcatheter arterial chemoembolization (TACE) can predict the anti-tumor effect of subsequent TACE with miriplatin in patients with recurrent hepatocellular carcinoma. Oncology 80: 188-194, 2011.

8. Okusaka T, Kasugai H, Shioyama Y, Tanaka K, Kudo M, Saisho H, et al: Transarterial chemotherapy alone versus transarterial chemoembolization for hepatocellular carcinoma: a randomized phase III trial. J Hepatol 51: 1030-1036, 2009.

9. Hu HT, Kim JH, Lee LS, Kim KA, Ko GY, Yoon HK, et al: Chemoembolization for hepatocellular carcinoma: multivariate analysis of predicting factors for tumor response and survival in a 362-patient cohort. J Vasc Interv Radiol 22: 917-923, 2011.

10. Levine B and Kroemer G: Autophagy in the pathogenesis of disease. Cell 132: 27-42, 2008.

11. Kondo Y, Kanzawa T, Sawaya R and Kondo S: The role of autophagy in cancer development and response to therapy. Nat Rev Cancer 5: 726-734, 2005.

12. Gao M, Yeh PY, Lu YS, Hsu CH, Chen KF, Lee WC, et al: OSU-03012, a novel celecoxib derivative, induces reactive oxygen species-related autophagy in hepatocellular carcinoma. Cancer Res 68: 9348-9357, 2008.

13. Huang X, Bai HM, Chen L, Li B and Lu YC: Reduced expression of LC3B-II and Beclin 1 in glioblastoma multiforme indicates a down-regulated autophagic capacity that relates to the progression of astrocytic tumors. J Clin Neurosci 17: 1515-1519, 2010.

14. Hamacher-Brady A, Brady NR and Gottlieb RA: Enhancing macroautophagy protects against ischemia/reperfusion injury in cardiac myocytes. J Biol Chem 281: 29776-29787, 2006.

15. Katavetin P, Miyata T, Inagi R, Tanaka T, Sassa R, Ingelfinger JR, et al: High glucose blunts vascular endothelial growth factor response to hypoxia via the oxidative stress-regulated hypoxiainducible factor/hypoxia-responsible element pathway. J Am Soc Nephrol 17: 1405-1413, 2006.
16. Matsui Y, Takagi H, Qu X, Abdellatif M, Sakoda H, Asano T, et al: Distinct roles of autophagy in the heart during ischemia and reperfusion: roles of AMP-activated protein kinase and Beclin 1 in mediating autophagy. Circ Res 100: 914-922, 2007.

17. Qiu W, Zhou B, Chu PG, Luh F and Yen Y: The induction of growth arrest DNA damage-inducible gene 45 beta in human hepatoma cell lines by S-adenosylmethionine. Am J Pathol 171: 287-296, 2007.

18. Qiu W, Zhou B, Zou H, Liu X, Chu PG, Lopez R, et al: Hypermethylation of growth arrest DNA damage-inducible gene 45 beta promoter in human hepatocellular carcinoma. Am J Pathol 165: 1689-1699, 2004.

19. Martin SK, Diamond P, Gronthos S, Peet DJ and Zannettino AC: The emerging role of hypoxia, HIF-1 and HIF-2 in multiple myeloma. Leukemia 25: 1533-1542, 2011.

20. Chen JL, Lin HH, Kim KJ, Lin A, Forman HJ and Ann DK: Novel roles for protein kinase Cdelta-dependent signaling pathways in acute hypoxic stress-induced autophagy. J Biol Chem 283: 34432-34444, 2008.

21. Karim MR, Kanazawa T, Daigaku Y, Fujimura S, Miotto G and Kadowaki M: Cytosolic LC3 ratio as a sensitive index of macroautophagy in isolated rat hepatocytes and H4-II-E cells. Autophagy 3: 553-560, 2007.

22. Kabeya Y, Mizushima N, Ueno T, et al: LC3, a mammalian homologue of yeast Apg8p, is localized in autophagosome membranes after processing. EMBO J 19: 5720-5728, 2000.

23. Olivé M, van Leeuwen FW, Janué A, Moreno D, TorrejónEscribano B and Ferrer I: Expression of mutant ubiquitin $\left(\mathrm{UBB}^{+1}\right)$ and p62 in myotilinopathies and desminopathies. Neuropathol Appl Neurobiol 34: 76-87, 2008.

24. Kim KR, Moon HE and Kim KW: Hypoxia-induced angiogenesis in human hepatocellular carcinoma. J Mol Med 80: 703-714, 2002.

25. Gwak GY, Yoon JH, Kim KM, Lee HS, Chung JW and Gores GJ: Hypoxia stimulates proliferation of human hepatoma cells through the induction of hexokinase II expression. J Hepatol 42: 358-364, 2005.

26. Sakdee JB, White RR, Pagonis TC and Hauschka PV: Hypoxiaamplified proliferation of human dental pulp cells. J Endod 35: 818-823, 2009.

27. Fukuda R, Zhang H, Kim JW, Shimoda L, Dang CV and Semenza GL: HIF-1 regulates cytochrome oxidase subunits to optimize efficiency of respiration in hypoxic cells. Cell 129: 111-122, 2007.

28. Koike M, Shibata M, Tadakoshi M, Gotoh K, Komatsu M, Waguri S, et al: Inhibition of autophagy prevents hippocampal pyramidal neuron death after hypoxic-ischemic injury. Am J Pathol 172: 454-469, 2008.

29. Hein S, Arnon E, Kostin S, Schönburg M, Elsässer A, Polyakova V, et al: Progression from compensated hypertrophy to failure in the pressure-overloaded human heart: structural deterioration and compensatory mechanisms. Circulation 107: 984-991, 2003.

30. Bristow RG and Hill RP: Hypoxia and metabolism. Hypoxia, DNA repair and genetic instability. Nat Rev Cancer 8: 180-192, 2008.

31. Shintani T and Klionsky DJ: Autophagy in health and disease: a double-edged sword. Science 306: 990-995, 2004.

32. Apel A, Zentgraf H, Buchler MW and Herr I: Autophagy - A double-edged sword in oncology. Int J Cancer 125: 991-995, 2009.

33. Komatsu M, Waguri S, Koike M, Sou YS, Ueno T, Hara T, et al: Homeostatic levels of p62 control cytoplasmic inclusion body formation in autophagy-deficient mice. Cell 131: 1149-1163, 2007.

34. Mathew R, Karp CM, Beaudoin B, Vuong N, Chen G, Chen HY, et al: Autophagy suppresses tumorigenesis through elimination of p62. Cell 137: 1062-1075, 2009.

35. Pursiheimo JP, Rantanen K, Heikkinen PT, Johansen T and Jaakkola PM: Hypoxia-activated autophagy accelerates degradation of SQSTM1/p62. Oncogene 28: 334-344, 2009.

36. Tasdemir E, Chiara Maiuri M, Morselli E, Criollo A,D'Amelio M, Djavaheri-Mergny M, et al: A dual role of p53 in the control of autophagy. Autophagy 4: 810-814, 2008.

37. Olovnikov IA, Kravchenko JE and Chumakov PM: Homeostatic functions of the p53 tumor suppressor: regulation of energy metabolism and antioxidant defense. Semin Cancer Biol 19: 32-41, 2009.

38. Karna P, Zughaier S, Pannu V, Simmons R, Narayan S and Aneja R: Induction of reactive oxygen species-mediated autophagy by a novel microtubule-modulating agent. J Biol Chem 285: 18737$18748,2010$. 